\title{
Metastin has potential as a suitable biomarker and novel effective therapy for cancer metastasis (Review)
}

\author{
SUNAO SHOJI $^{1}$, XIAN YANG TANG ${ }^{2}$, HARUHIRO SATO $^{3}$, YUKIO USUI $^{1}$, \\ TOYOAKI UCHIDA $^{1}$ and TOSHIRO TERACHI ${ }^{1}$
}

Departments of ${ }^{1}$ Urology, ${ }^{2}$ Pathology, and ${ }^{3}$ Medicine, Tokai University School of Medicine, Kanagawa, Japan

Received May 4, 2010; Accepted July 6, 2010

DOI: 10.3892/ol_00000136

\begin{abstract}
Cancer metastasis is a leading cause of death in cancer patients and is a multistep process involving complex interactions between tumor and host cells. To metastasize, tumor cells must invade or migrate from the primary tumor and be transported to close or distant secondary sites. A tumor cell should successfully accomplish each step of the pathway or metastasis may not develop. KiSS-1 is a human metastasis suppressor gene that inhibits metastasis of human melanomas and breast carcinomas without affecting tumorigenicity. KiSS-1 encodes a carboxy-terminally amidated peptide with 54 aminoacid residues. The peptide was isolated from human placenta as the endogenous ligand of an orphan G-protein-coupled receptor and termed 'metastin'. The literature reports metastin related to human carcinoma, such as melanoma, thyroid cancer, esophageal squamous cell carcinoma (ESCC), hepatocellular carcinoma, pancreatic carcinoma, as well as breast, ovarian, bladder and kidney cancer. These malignancies are difficult to treat and, even in early-stage cancer, a number of patients develop metastasis shortly after surgery. Studies have suggested that metastin inhibits tumor invasion or migration through focal adhesion kinase, paxillin, MAP kinase or Rho A. Additionally, metastin may be a biomarker in ESCC, pancreatic carcinoma and bladder cancer. Metastin has potential as a suitable biomarker in the identification of tumors with high metastatic potential and as a novel effective treatment modality for patients with metastasis.
\end{abstract}

\section{Contents}

1. Introduction

2. Identification and basement study of metastin related to cancer metastasis

Correspondence to: Dr Sunao Shoji, Department of Urology, Tokai University School of Medicine, Shimokasuya 143, Isehara, Kanagawa 259-1193, Japan

E-mail: sunashoj@mail.goo.ne.jp

Abbreviations: ESCC, esophageal squamous cell carcinoma; HCC, hepatocellularcarcinoma; OLT, orthotopic liver transplantation; $\mathrm{RCC}$, renal cell carcinoma; UC, urothelial carcinoma

Key words: KiSS-1, metastin, metastin receptor, cancer metastasis
3. Metastin inhibits chemotaxis and invasion of the metastin receptor-transfected $\mathrm{CHO}$ cells in vitro and attenuates the pulmonary metastasis of metastin receptor-transfected B16-BL6 melanoma in vivo

4. Metastin receptors are overexpressed in papillary thyroid cancer tissues and activate MAP kinase in thyroid cancer cells

5. Metastin receptors, a promising target for the suppression of metastasis and metastin, a potential biomarker for predicting cancer presence and progression in pancreatic cancer

6. Metastin/metastin receptor system in hepatocellular carcinoma and breast cancer is influenced by hormonal disorders

7. Metastin and metastin receptor signals may suppress the invasive phenotype of epithelial ovarian cancer

8. Loss of metastin or metastin receptor mRNA expression is an important biomarker for detecting lymph node metastasis in esophageal squamous cell carcinoma

9. Expression levels of metastin mRNA provide prognostic information in bladder carcinoma

10. Metastin inhibits the migration and invasion of renal cell carcinoma via overexpression of the metastin receptors

11. Conclusion

\section{Introduction}

Cancer metastasis is a leading cause of death in cancer patients and is a multistep process involving complex interactions between tumor and host cells. To metastasize, tumor cells must invade from the primary tumor by dissociating from the tumor mass and be transported to close or distant secondary sites. Single cells, homotypic clusters of cells or heterotypic emboli then remain at the secondary site and use organspecific and organ non-specific mechanisms which invade the surrounding tissue and respond to growth signals. A tumor cell should successfully accomplish each step in the pathway or metastasis may not develop. Positive and negative regulators exist for each step in the metastatic cascade, implying the involvement of numerous different genes.

KiSS-1 is a human metastasis suppressor gene that inhibits the metastasis of human melanomas and breast carcinomas, without affecting tumorigenicity (1). KiSS-1 encodes a carboxy-terminally amidated peptide with 54 amino-acid residues. The peptide was isolated from human placenta as the endogenous ligand of an orphan G-protein-coupled receptor (metastin receptor, hOT7T175) and was termed 'metastin' (2). 
Ohtaki et al reported that metastin inhibits the chemotaxis and invasion of metastin receptor-transfected $\mathrm{CHO}$ cells in vitro and attenuates the pulmonary metastasis of metastin receptortransfected B16-BL6 melanomas in vivo (2). Results suggest the potential action mechanisms of KiSS-1 and a novel therapeutic approach. Since the report by Ohtaki et al, metastin has been considered an agent with the potential to inhibit cancer metastasis, resulting in the investigation of various cancer metastases. The literature currently covers melanoma (2), thyroid cancer (3), pancreatic cancer $(7,8)$, hepatocellular carcinoma (HCC) $(5,6)$, breast cancer $(9)$, ovarian cancer $(10)$, esophageal squamous cell carcinoma (ESCC) (4), bladder cancer $(11,12)$ and kidney cancer (13). Additionally, it has been suggested that metastin inhibits tumor invasion or migration through focal adhesion kinase, paxillin, MAP kinase or Rho A $(2,3,13,14)$.

\section{Identification and basement study of metastin related to cancer metastasis}

Lee et al identified various candidate metastasis-suppressor cDNAs by subtractive hybridization and differential display by comparing human metastatic melanoma cell lines (C8161) and non-metastatic hybrid clones (neo6/C8161.1 cells). One clone, termed KiSS-1, was expressed only in neo6/C8161.1 cells. KiSS-1 cDNA has a single open reading frame that encodes a protein of 164 amino acids with a predicted molecular mass of $18 \mathrm{kDa}(1)$. Ohtaki et al found a rat orphan receptor (rOT7T175) that was nearly identical to GPR54 during a search for novel G-protein-coupled receptors (GPCRs) using a degenerate polymerase chain reaction (PCR) strategy. To identify the endogenous ligand of a human orphan receptor, these authors established a stable $\mathrm{CHO}$ cell line expressing the human counterpart metastin receptor (CHO/h175). Although hOT7T175 had 39.2\% amino-acid identity to human galanin receptor GALR2, the cells did not show any response to a panel of known peptides, including galanin and galanin-like peptides. On the other hand, the human placental extract induced a robust increase in the intracellular calcium ion concentration $\left(\left[\mathrm{Ca}^{2+}\right] \mathrm{i}\right)$ in $\mathrm{CHO} / \mathrm{h} 175$ cells. The amino-terminal sequence obtained for the isolated peptide was identical to the partial amino-acid sequence (Gly 68 to Ala 88) of the KiSS-1 gene product. The sequence was isolated from human placenta as the endogenous ligand of an orphan G-proteincoupled receptor and was termed 'metastin' (2).

Matrix metalloprotease (MMP) plays an important role in development and morphogenesis by participating in extracellular matrix re-modeling (15). Cancer cells also utilize MMP for invasion and metastasis. Invading cells are forced to proliferate within an embedded dense three-dimensional matrix composed largely of type I collagen or cross-linked fibrin (16). Takino et al reported that metastin forms a complex with pro-MMP and active MMP-2, while MMP-9, matrix type (MT) 1-MMP, MT3-MMP and MT5-MMP cleaved the Gly118-Leu119 peptide bond of both full-length metastin and metastin decapeptide (17). Metastin decapeptide induced the formation of focal adhesion and actin stress fibers in cells expressing the receptor. Moreover, digestion of the metastin decapeptide by MMP abolished its ligand activity. Takino et al proposed that i) metastin be used as an antimetastatic agent in combination with the MMP inhibitor, or ii) MMP-resistant forms of metastin be developed that may also be efficacious.

Metastin has attracted cancer investigations due to its novelty and potential to inhibit cancer metastasis. Subsequently, the pertinent literature related to metastin and cancer metastasis is reviewed herein.

\section{Metastin inhibits chemotaxis and invasion of the metastin receptor-transfected $\mathrm{CHO}$ cells in vitro and the attenuates pulmonary metastasis of metastin receptor-transfected B16-BL6 melanoma in vivo}

Ohtaki et al studied whether metastin suppresses tumor metastasis (2). These investigators examined the effect of peptide on cell motility, a characteristic that is considered crucial for the invasion of cancer cells. Metastin inhibited the chemotaxis of metastin receptor-transfected $\mathrm{CHO}(\mathrm{CHO} / \mathrm{h} 175)$ cells towards fetal calf serum, but did not affect that of $\mathrm{CHO}$ mock transfectants (CHO/mock). In an in vitro invasion assay that tested migration through a Matrigel-coated porous filter, metastin inhibited the invasion of $\mathrm{CHO} / \mathrm{h} 175$ cells specifically with an $\mathrm{IC}_{50}$ value of $\sim 50 \mathrm{nM}$. Metastin inhibited the fibronectin-induced chemotaxis of the metastin receptor-transfected B16-BL6 mouse melanoma (B16-BL6/h175) cells, but not that of mocktransfected melanoma (B16-BL6/mock) cells. Furthermore, Ohtaki et al found that metastin induced the excessive formation of focal adhesions (vinculin-positive patches) and stress fibers in B16-BL6/h175 cells (2). The cells began to express focal adhesions 5 min after the addition of metastin and the majority of cells expressed focal adhesions densely after $30 \mathrm{~min}$. Excessive stress fibers were formed after $30 \mathrm{~min}$, but not after $5 \mathrm{~min}$. Metastin also induced the phosphorylation of focal adhesion kinase (FAK) and paxillin, which is believed to be essential for the formation of focal adhesions. The phosphorylation of FAK occurred rapidly and almost ceased after $10 \mathrm{~min}$, whereas that of paxillin lasted longer. The same authors hypothesized that metastin attenuates cellular motility by excessively inducing adhesive phenotypes (2). In spontaneous pulmonary metastasis assays, metastin-treated mice transplanted with B16-BL16/h175 cells showed a marked decrease in the number of metastasized tumor foci compared to mice that had received a vehicle. On the other hand, metastin did not affect the metastasis of B16-BL6/ mock melanomas. Therefore, the observed effect on tumor metastasis is likely due to the direct action of metastin on tumor cells, although metastin may have also exerted pharmacological effects on host mice.

\section{Metastin receptors are overexpressed in papillary thyroid cancer tissue and activate MAP kinase in thyroid cancer cells}

Ringel et al analyzed thyroid tissue samples obtained at the time of surgery from 23 patients with thyroid cancer (10 with follicular and 13 with papillary cancer) and from 2 patients with benign, non-functioning follicular adenomas (3). The expression of metastin receptor mRNA levels, measured using real-time RT-PCR, was detected in 10 of 13 papillary thyroid cancers and in only 2 of 10 follicular thyroid cancer types. Statistically, papillary carcinomas were more likely to express metastin receptors than either follicular carcinoma or normal 
thyroid tissue. In paired samples, metastin receptor expression was overexpressed in the papillary cancers with adjacent normal tissue specimens. A thyroid cancer cell line (ARO) that expresses a metastin receptor was analyzed in order to evaluate the signaling pathways activated by endogenous metastin receptors. It was found that metastin increased ERK, but not Akt or p38 activation, in a dose-dependent manner under these conditions.

\section{Metastin receptors, a promising target for the suppression of metastasis and metastin, a potential biomarker for predicting cancer presence and progression in pancreatic cancer}

Masui et al reported that KiSS-1 expression is suppressed and metastin receptor expression is enhanced in pancreatic cancer tissue when compared to that in normal tissue (18). Exogenous metastin was reported not to suppress cell proliferation, but significantly reduce the in vitro migration of the pancreatic cancer cell line (PANC-1) and induce the activation of ERK1 in two pancreatic cancer cell lines (PANC-1 and AsPC-1) (18). In addition, three novel short variant forms of metastin, i.e., FM053a2TFA, FM059a2TFA and FM052a4TFA, were synthesized. These metastin variants significantly suppressed the migration of PANC-1 cells and activated ERK1. The results suggested that metastin receptors are a promising target for the suppression of metastasis.

Katagiri et al reported plasma metastin in pancreatic cancer patients (8). Blood samples from 24 healthy volunteers, as well as 33 patients with pathologically confirmed pancreatic cancer, were analyzed prior to the latter patients being treated with chemotherapy, radiation or surgery. Peptide levels in plasma were measured using highly sensitive EIAs for metastin-LI. Plasma metastin-LI levels also showed no significant correlation with tumor markers CEA, CA19-9, CA125 or DUPAN-2. Moreover, levels in pancreatic cancer patients were significantly higher than those in healthy volunteers of a similar age. Notably, plasma metastin-LI levels in patients in T3, T4, N0, M0 and M1 groups were significantly higher than those in healthy volunteers, although plasma metastin-LI levels in patients with T2 tumors were not significantly different from those in healthy volunteers. Plasma metastin-LI levels in patients did not significantly differ by UICC (International Union Against Cancer) TNM classification (6th edition) (19). Therefore, Katagiri et al hypothesized that a high level of metastin in pancreatic cancer patients is secreted from normal tissue as a reaction to cancer progression, as a self-defense mechanism. Although further prospective studies are required, metastin has potential as a biomarker for predicting cancer presence and progression (8).

\section{Metastin/metastin receptor system in hepatocellular carcinoma and breast cancer is influenced by hormonal disorders}

Ikeguchi et al analyzed the expression of metastin and metastin receptor mRNA levels in HCC using real-time RT-PCR (5). Tissue samples were obtained from 60 patients with HCC who had received surgical hepatectomy, but not pre-operative chemotherapy or radiation therapy. Additionally, 8 normal liver tissues were obtained from 8 patients without HCC or liver cirrhosis. It was reported that although the average level of metastin mRNA did not change among normal and non-cancerous cirrhotic livers and carcinomas, a significantly high level of metastin mRNA expression was detected in 13 carcinomas (5). Moreover, the percentage of the positive expression of metastin receptor mRNA significantly increased from $12.5 \%$ in normal livers and $26.7 \%$ in non-cancerous cirrhotic livers to $48.3 \%$ in the carcinomas. The tumors with overexpression of metastin and metastin receptor mRNA were in an advanced stage. Ikeguchi et al concluded that the overexpressed metastin and metastin receptor mRNA was frequently observed in HCC, and it was suggested that overexpressed metastin and metastin receptor peptides mediate growth signals into cancer cells in HCCs (5). Schmid et al analyzed samples from 142 patients who underwent orthotopic liver transplantation (OLT) for HCC by immunohistochemistry (6). Metastin receptor immunoreactivity was correlated with the pathological tumor grade and stage. These authors showed, by multivariate analysis, that an intense metastin expression in $\mathrm{HCC}$ was associated with the poor prognosis of patients undergoing OLT.

Martin et al reported that frozen sections from breast cancer primary tumors (matched tumor 124 and background 33) were immunostained with metastin antibody (9). The results of real-time RT-PCR showed that the expression levels of metastin were higher in tumors compared to background tissues and were significantly increased in nodepositive tumors compared to node-negative ones. The mRNA of metastin expression was also elevated with increasing grade and TNM status, but no such trends were noted with the metastin receptor. The expression of metastin mRNA was higher in patients who had succumbed to breast cancer than in those who had remained healthy, whereas the expression of the receptor showed a decrease in the two groups. Using immunohistochemistry, the expression of metastin was higher in tumor sections than in normal tissues. The insertion of metastin mRNA into the human breast cancer cell line MDA-MB-231 resulted in cells that were significantly more motile and invasive in behavior, with reduced adhesion to the matrix, using respective assays. Thus, metastin showed an increase in human breast cancer, particularly in patients with aggressive tumors and fatal disease. The overexpression of metastin was therefore correlated with poor prognosis in patients with breast cancer, and acted as a possible metastasis promoter in human breast cancer cells in vitro. This study showed that metastin may have a potential role as a tumor suppressor in breast cancer and that future studies should include in vivo models.

By contrast, recently published data on metastin and/or metastin receptor mRNA expression in other human malignancies reported a strong correlation between metastin and/ or metastin receptor mRNA overexpression and an improved clinical course $(2-4,7,9)$. The mechanisms regarding the reason for the expression of metastin mRNA product showing an opposite impact on the clinical outcome of $\mathrm{HCC}$ and breast cancer compared to other malignant tumors remain to be elucidated. Ikeguchi et al postulated that elevated estrogen levels 


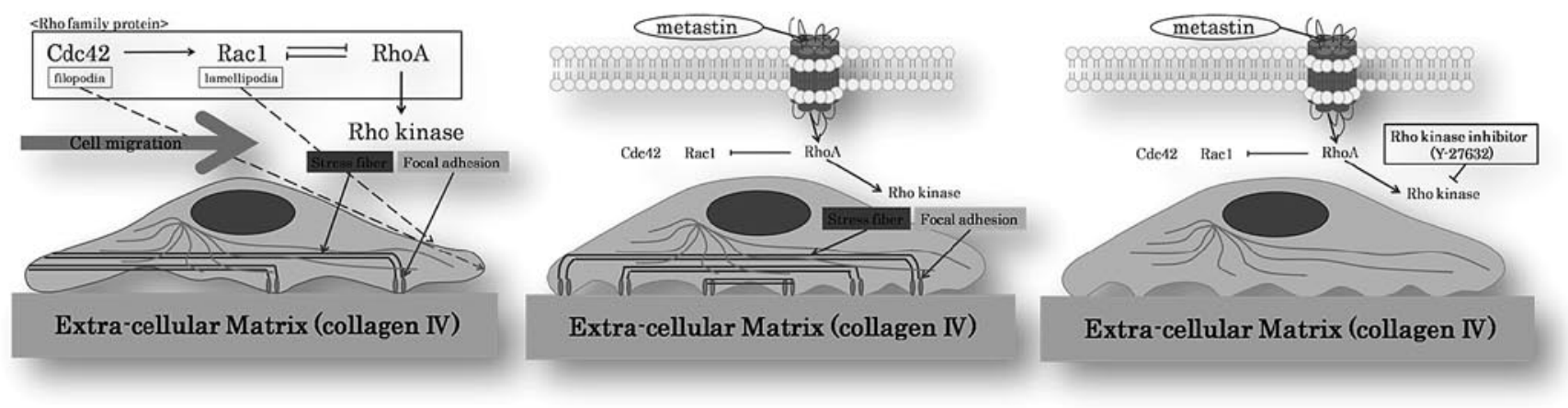

Figure 1. Relationship between Rho family protein and metastin. Rho regulates cell adhesion and motility by sequence activation of its family members, i.e., Cdc $42 \rightarrow$ Racl $\Leftrightarrow$ RhoA GTPase. (A) This sequence is associated with time-dependent changes in cell morphology from filopodia/actin spikes to lamellipodia/ membrane ruffles to stress fibers. (B) The present results clearly show that metastin changed the shape of RCC cells through this Rho-GTPase route by activating one or a number of its members. (C) This reaction was blocked when Caki-1 and ACHN were pre-treated with the pharmacological Rho kinase inhibitor Y-27632.

modulate the expression patterns of metastin (5). Patients with HCC exhibit liver cirrhosis and are therefore prone to a hyper-estrogenic state. The fact that metastin/metastin receptor systems in HCC and breast cancer may be influenced by hormonal disorders is supported by the findings of Muir and Ohtaki, who reported that the plasma level of metastin was 10,000 -fold higher in pregnant than in non-pregnant women $(2,20)$. However, further studies are required to clarify the possible link between estrogen signaling and metastin and metastin receptor expression in tumors.

7. Metastin and metastin receptor signals suppress the invasive phenotype of epithelial ovarian cancer

Hata et al analyzed the expression levels of metastin, the metastin receptor gene, using real-time RT-PCR in 76 epithelial ovarian cancer surgical specimens (10). These authors reported that the presence of residual tumor was negatively associated with metastin and metastin receptor gene expression, and that patient age at diagnosis was significantly associated with metastin gene expression. It was also found that the prognosis of patients with a low expression of the metastin receptor gene was significantly worse than that of those with a high expression (10). However, metastin gene expression had no impact on patient survival, but a combination of metastin and metastin receptor gene expression significantly affected patient prognosis. The results provided new insight into the biological behavior of epithelial ovarian cancer. Furthermore, metastin and metastin receptor signals may suppress the invasive phenotype of epithelial ovarian cancer.

\section{Loss of metastin or metastin receptor mRNA expression may be an important biomarker for detecting lymph node metastasis in esophageal squamous cell carcinoma}

Ikeguchi et al reported on the mRNA levels of metastin and metastin receptor in tumors and non-cancerous epithelia of 71 patients with ESCC who underwent surgical esophageal resection using real-time RT-PCR (5). The mRNA levels of metastin and metastin receptor were analyzed quantitatively using realtime RT-PCR and compared to the clinical findings. The mean metastin and metastin receptor levels of the tumors were similar to those in non-cancerous epithelia. Loss of metastin and metastin receptor mRNA was detected in 38 and $61 \%$ of tumors, respectively. Ikeguchi et al compared disease-specific survival among three groups (group A: 17 patients with tumors with preserved expression of both metastin and metastin receptor mRNA; group B: 34 patients with tumors with loss of one of the two mRNA and group C: 14 patients with tumors with loss of metastin and metastin receptor mRNA) (4). The disease-specific 5-year survival rates were 68,31 and $32 \%$ in groups $\mathrm{A}, \mathrm{B}$ and $\mathrm{C}$, respectively. To compare differences in the survival curves between group A and groups B and C, the depth of tumor invasion and lymph node metastasis were detected as strong prognostic factors in a multivariate survival analysis. Loss of metastin or metastin receptor mRNA expression may therefore be an important biomarker for detecting lymph node metastasis in ESCC.

\section{Expression levels of metastin mRNA provide prognostic information in bladder carcinoma}

Nicolle et al analyzed the expression of metastin and metastin receptor mRNA levels in urothelial carcinoma (UC) using real-time RT-PCR (12). It was reported that in UC, from bladder specimens, the expression levels of metastin mRNA showed a significant increase (carcinoma of low grade $<$ high grade) compared to normal bladder tissue (12). The expression levels of metastin receptor mRNA also showed high expression levels in bladder carcinomas compared to normal bladder tissue and the difference between low- and high-grade groups was significant (low grade $<$ high grade). When the expression levels were classified (low, normal and high), a correlation was found between increased aggressiveness of the tumor ranges and the expression levels of the metastin receptor.

Using in situ hybridization, Sanchez-Carbayo et al reported a low expression of metastin in 34\% and a high expression of metastin in $45 \%$ of high-grade bladder cancers. No correlation was found between the expression levels of metastin and the metastin receptor, respectively. These investigators also suggested that the expression of metastin mRNA provided prognostic information. Furthermore, complete loss of 
metastin mRNA was noted in the invasive tumors of that study as compared to their respective normal urothelium. The expression of metastin mRNA was found to be significantly correlated with the histopathological stage. Patients with a lower expression of metastin mRNA showed a direct correlation with overall survival in a subset of bladder tumors whose follow-up was available $(n=69)$ (11). A potential tumor suppressor role in bladder cancer was shown for metastin. Moreover, a predictive value was observed by identifying the patients with a poor outcome.

\section{Metastin inhibits the migration and invasion of renal cell carcinoma via overexpression of the metastin receptors}

We reported the mRNA levels of metastin and metastin receptors in tumor and normal tissues from fresh tissues of renal cell carcinomas (RCCs) (matched tumor 25 and background 25) (13). The results showed that the metastin receptors are highly expressed in RCCs. Our results present the possibility that metastin receptors are a target for molecular therapy in the inhibition of RCC metastasis and local invasion due to the high affinity between metastin and its receptor. In the literature, the prognosis for RCC based on histological subtypes and 5-year cancer-specific survival for clear (conventional) cell carcinoma is shorter than that for papillary and chromophobe carcinoma (21). Although our 25 RCC cases did not include all three (clear cell type, papillary type and unclassified) of these subtypes, no differences in metastin and/or its receptor between clear-cell and papillary carcinoma were found. Another notable finding presented in this study is that metastin induced the excessive formation of focal adhesions, which are located at the ends of stress fibers in RCC cell lines (Caki-1 and ACHN cells) (Fig. 1B). This reaction was blocked when we pre-treated Caki-1 and ACHN with the pharmacological Rho kinase inhibitor Y-27632 (Fig. 1C). However, the mechanism of how intracellular signals induce excessive focal adhesion through the metastin receptor has yet to be elucidated. In light of previous investigations, we hypothesized that metastin changes the shape of RCC cells through this Rho-GTPase pathway by activating one or a number of its members. Using Caki-1, it was found that the expression of the von Hippel-Lindau tumor suppressor protein (pVHL) was up-regulated by hypoxia through RhoA-dependent activity and that Rho kinase inhibitor Y-27632 inhibits this pVHL induction by hypoxia (22). Rho kinase is involved in the formation of stress fibers and focal adhesion in fibroblasts. Numerous observations suggest that RhoA regulates the formation of focal adhesion associated with stress fibers by activating myosin, whereas $\mathrm{Cdc} 42 / \mathrm{Rac} 1$ regulates the formation of focal complexes associated with filopodia and lamellipodia, and disassembles focal adhesion and stress fibers through PAK. Rho regulates cell adhesion and motility by sequence activation of its family members, i.e., Cdc $42 \rightarrow$ Rac $1 \Leftrightarrow$ RhoA GTPase. This sequence is associated with time-dependent changes in cell morphology from filopodia/actin spikes to lamellipodia/ membrane ruffles to stress fibers (Fig. 1A). Each member of the Rho-GTPase family has its specific intracellular signal. Our current results clearly show that metastin changes the shape of RCC cells through this Rho-GTPase pathway by activating one or a number of its members (Fig. 1B). This phenomenon may provide fundamental knowledge for exploiting new treatments for RCC cases. Cdc42/Racl and RhoA exert mutually antagonistic effects at some point in the process of contact initiation, maturation and turnover. The balance of activities between the Rho-GTPase family and downstream signaling of Rho determines the final patterns of adhesion and cytoskeleton organization. In addition, a phenomenon exists whereby a high concentration of Cdc42 suppresses membrane ruffling, which is dependent on Rac1. We did not find any disruption of microtubules, as evidenced by tubulin staining in metastin-supplied cells. This phenomenon is consistent with the finding that metastin has no effect on Caki-1 and ACHN cell proliferation. It is known that there is a pathway separate from the that leading to cell adhesion, which mediates the growth-promoting action of Rho (23). Although the detailed mechanism should be investigated further, it is speculated that metastin may have inhibited cell migration and invasion by regulating Rho kinase in RCC. It is known that the metastasis and invasion of RCC involve various factors. Thus, the pathways associated with metastin and its receptors are crucial.

\section{Conclusion}

In conclusion, it is evident that further research is necessary before the precise role and effect of metastin and the metastin receptor in human cancer can be elucidated. However, since cancer metastasis is difficult to treat, the results of basic and clinical studies of metastin are noteworthy. Metastin should be further investigated for the future treatment of cancer.

\section{References}

1. Lee JH, Miele ME, Hicks DJ, et al: KiSS-1, a novel human malignant melanoma metastasis-suppressor gene. J Natl Cancer Inst 88: 1731-1737, 1997.

2. Ohtaki T, Shintani Y, Honda S, et al: Metastasis suppressor gene KiSS-1 encodes peptide ligand of a G-protein-coupled receptor. Nature 31: 613-617, 2001.

3. Ringel MD, Hardy E, Bernet VJ, et al: Metastin receptor is overexpressed in papillary thyroid cancer and activates MAP kinase in thyroid cancer cells. J Clin Endocrinol Metab 87: 2399, 2002

4. Ikeguchi M, Yamaguchi K and Kaibara N: Clinical significance of the loss of KiSS-1 and orphan G-protein-coupled receptor (hOT7T175) gene expression in esophageal squamous cell carcinoma. Clin Cancer Res 10: 1379-1383, 2004.

5. Ikeguchi M, Hirooka Y and Kaibara N: Quantitative reverse transcriptase polymerase chain reaction analysis for KiSS-1 and orphan G-protein-coupled receptor (hOT7T175) gene expression in hepatocellular carcinoma. J Cancer Res Clin Oncol 129: 531-535, 2003.

6. Schmid K, Wang X, Haitel A, et al: KiSS-1 overexpression as an independent prognostic marker in hepatocellular carcinoma: an immunohistochemical study. Virchows Arch 450: 143-149, 2007.

7. Nagai K, Doi R, Katagiri F, et al: Prognostic value of metastin expression in human pancreatic cancer. J Exp Clin Cancer Res 28: 9, 2009.

8. Katagiri F, Nagai K, Kida A, et al: Clinical significance of plasma metastin level in pancreatic cancer patients. Oncol Rep 21: 815-819, 2009.

9. Martin TA, Watkins G and Jiang WG: KiSS-1 expression in human breast cancer. Clin Exp Metastasis 22: 503-511, 2005.

10. Hata K, Dhar DK, Watanabe Y, et al: Expression of metastin and a G-protein-coupled receptor (AXOR12) in epithelial ovarian cancer. Eur J Cancer 43: 1452-1459, 2007.

11. Sanchez-Carbayo M, Capodieci P and Cordon-Cardo C: Tumor suppressor role of KiSS-1 in bladder cancer: loss of KiSS-1 expression is associated with bladder cancer progression and clinical outcome. Am J Pathol 162: 609-617, 2003. 
12. Nicolle G, Comperat E, Nicolaïew N, et al: Metastin (KISS-1) and metastin-coupled receptor (GPR54) expression in transitional cell carcinoma of the bladder. Ann Oncol 18: 605-607, 2007.

13. Shoji S, Tang XY, Umemura S, et al: Metastin inhibits migration and invasion of renal cell carcinoma with overexpression of metastin receptor. Eur Urol 55: 441-449, 2009.

14. Harms JF, Welch DR and Miele ME: KISS1 metastasis suppression and emergent pathways. Clin Exp Metastasis 20: 11-18, 2003.

15. Liotta LA, Thorgeirsson UP and Garbisa S: Role of collagenases in tumor cell invasion. Cancer Metastasis Rev 1: 277-288, 1982.

16. Fisher KE, Sacharidou A, Stratman AN, et al: MT1-MMP- and Cdc42-dependent signaling co-regulate cell invasion and tunnel formation in 3D collagen matrices. J Cell Sci 122: 4558-4569, 2009.

17. Takino $\mathrm{T}$, Koshikawa N, Miyamori $\mathrm{H}$, et al: Cleavage of metastasis suppressor gene product KiSS-1 protein/metastin by matrix metalloproteinases. Oncogene 22: 4617-4626, 2003.

18. Masui T, Doi R, Mori T, et al: Metastin and its variant forms suppress migration of pancreatic cancer cells. Biochem Biophys Res Commun 315: 85-92, 2004.
19. Sobin L and Witte KC (eds): Anonymous: Pancreas. In: TNM Classification of Malignant Tumours. Wiley-Lewis, New York, pp93-96, 2002.

20. Muir AI, Chamberlain L, Elshourbagy NA, et al: AXOR12, a novel human $G$ protein-coupled receptor, activated by the peptide KiSS-1. J Biol Chem 276: 28969-28975, 2001.

21. Teloken PE, Thompson RH, Tickoo SK, et al: Prognostic impact of histological subtype on surgically treated localized renal cell carcinoma. J Urol 182: 2132-2136, 2009.

22. Turcotte S, Desrosiers RR and Beliveau R: Hypoxia upregulates von Hippel-Lindau tumor-suppressor protein through RhoAdependent activity in renal cell carcinoma. Am J Physiol Renal Physiol 286: F338-F348, 2004.

23. Narumiya S: The small GTPase Rho: cellular functions and signal transduction. J Biochem 120: 215-218, 1996. 